\title{
Extra-cranial aneurysm of the internal carotid artery treated by proximal ligation
}

\author{
I. R. HAYWOOD \\ F.R.C.S.
}

\author{
HAROLD Ellis \\ D.M., M.Ch., F.R.C.S.
}

\section{Surgical Unit, Westminster Hospital, London}

\begin{abstract}
Summary
A case of aneurysm of the extra-cranial section of the internal carotid artery extending to the base of the skull is described. Treatment comprised proximal vessel ligation, the tolerance of the patient's cerebral circulation to this procedure first being assessed by measuring the back pressure across the circle of Willis after temporary carotid artery occlusion. The value of this technique is discussed.
\end{abstract}

\section{Introduction}

Although by 1926 Winslow had collected 106 recorded cases of extra-cranial carotid aneurysms, and Alexander, Wigser and Davis (1964) reviewed 166 cases in a presentation of the first reported case of bilateral aneurysms, extra-cranial carotid aneurysms are uncommon: for example, in a review (Beall et al., 1962) of 2300 aneurysms in peripheral vessels, presenting at the Mayo Clinic, only 7 were situated in the carotids.

Aneurysms may occur in either the external, internal or common carotid arteries but most papers deal with those of the internal, presumably because of the special problems in their management. Numerous aetiological factors have been cited similar to those causing aneurysms in other sites including trauma, arteriosclerosis, cystic medial necrosis and, in previous times, local infection in the neck appears to have played a part. In Winslow's paper the number of cases which appear to have been concomitant with scarlet fever is most striking. Congenital aneurysms in childhood as have been described at other sites, for example, in the brachial artery (Colin and Ellis, 1971), have not been reported in the carotids. However, congenital tissue defects have been noted in the tunica media of aneurysms of the circle of Willis by Carmichael (1950) and, lately, attention has been drawn to fibromuscular hyperplasia in the internal carotids as a cause of aneurysm by Bergentz et al. (1976). No case of syphilis has been blamed for carotid aneurysm. Sex incidence would appear to be equal.
In nearly all cases, presentation has been by a painless mass noticed in the neck and within the paratonsillar fossa in the mouth. Differential diag- of noses include a tortuous atherosclerotic carotid artery, carotid body tumour, and enlarged overlying lymph nodes. Often, distinction can be made only by angiography. Paratonsillar abscess was a common differential diagnosis in earlier days, the clinician's error becoming obvious on incision.

Sequelae may eventually arise from within, as a result of embolism-causing stroke; or from without, as a result of pressure (by expansion or rupture) on external structures. Hoarseness, dysę phagia and respiratory obstruction have all beet described in the latter situation and, occasionallyo catastrophic haemorrhage has occurred, usually $\vec{y}^{+}$ owing to injudicious incision as above, but also during endotracheal intubation and, in one case, after accidental perforation by a patient's pipe stem. Winslow (1926) noted that in 35 patients left untreated, the mortality from the natural history of the aneurysm was $71 \%$, all the deaths occurring within one year of the onset of symptoms, and often after only a few weeks. Surgical treatment is therefore mandatory, and most authorities consider excision of the aneurysm the treatment of choice where this is possible. Usually, only a short length of the vessel is involved. Reconstruction may be by direct end-toend anastomosis (Rydell and Jennings, 1956, described bridging a $4 \cdot 5-\mathrm{cm}$ defect by mobilizing the ends of the vessel), insertion of a Dacron prosthesis or vein graft, or by using the external carotid artery after ligation of its distal end. Thompson and Austin (1957) described wrapping of the aneurysm with fascia or cellophane but, although this might well control expansion, its value in preventing emboli is obviously doubtful.

\section{Case report \\ A 54-year-old spinster was initially admitted to the Lister Hospital, Stevenage. She had noticed a pain- less swelling in the left side of her neck which had}




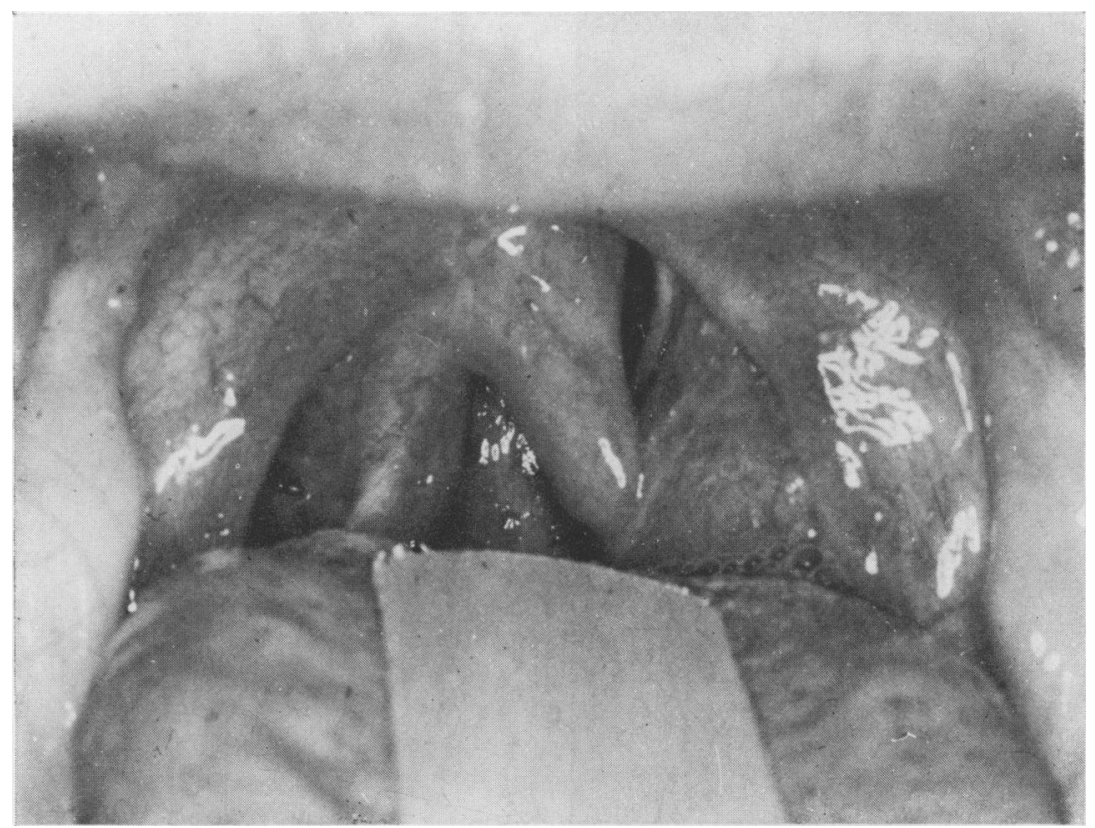

FIG. 1. View inside the mouth showing the mass in the left paratonsillar fossa which was pulsatile, pushing the uvula to the right.

gradually increased in size over the previous 14 months. It came to the attention of her medical attendant during routine follow-up examination for mammary carcinoma which had been treated by right simple mastectomy 7 years previously.

Examination showed a normotensive individual in good general health. There was no evidence of recurrence of her breast carcinoma. A pulsatile mass, behind the angle of the mandible, $3 \times 5 \mathrm{~cm}$ in diameter was noted and, within one month, the mass was found to extend into the paratonsillar fossa, pushing the tonsil to the midline (Fig. 1).

\section{Investigations}

An angiogram by needle puncture of the common carotid artery had been performed at the Lister Hospital and showed an aneurysm of the internal carotid artery extending from a point $2 \mathrm{~cm}$ distal from the carotid bifurcation to the base of the skull (Fig. 2). The external carotid artery was displaced but otherwise normal. Haemoglobin, white blood count, chest X-ray, spirochaete serology and ECG were all normal.

Surgery was considered essential but excision impractical so the decision was made to perform proximal ligation. Operation (H.E.) was performed under local anaesthesia, the patient being sedated with diazepam but alert. The carotid bifurcation was exposed. The external carotid artery was occluded and the pressure measured in the common carotid artery using a needle and transducer, and found to be $120 \mathrm{mmHg}$. The common carotid artery was then clamped below the needle and the pressure was found to fall to $65 \mathrm{mmHg}$, this representing the back pressure in the internal carotid artery across the circle of Willis. The patient tolerated occlusion for 5 min without any subjective or objective signs of cerebral ischaemia. The internal carotid artery was then ligated in continuity just beyond the bifurcation.

Postoperatively it was noted that all pulsation in the aneurysm had ceased. She was nursed in a horizontal position for one week and then allowed to sit up slowly. On the first day of altered posture she developed a mild right hemiparesis with voice change. She was immediately returned to the horizontal position and within $24 \mathrm{hr}$ her neurological status had returned to normal. A more gradual return to the vertical was initiated only after 4 more weeks in bed. She had suffered no further sequelae up to the time of review 6 months after operation.

\section{Discussion}

Treatment by excision and reconstruction was not considered feasible in this patient since the aneurysm extended to the base of the skull and this would have produced the problems of extensive dissection, control of the distal vessel during surgery and repair 


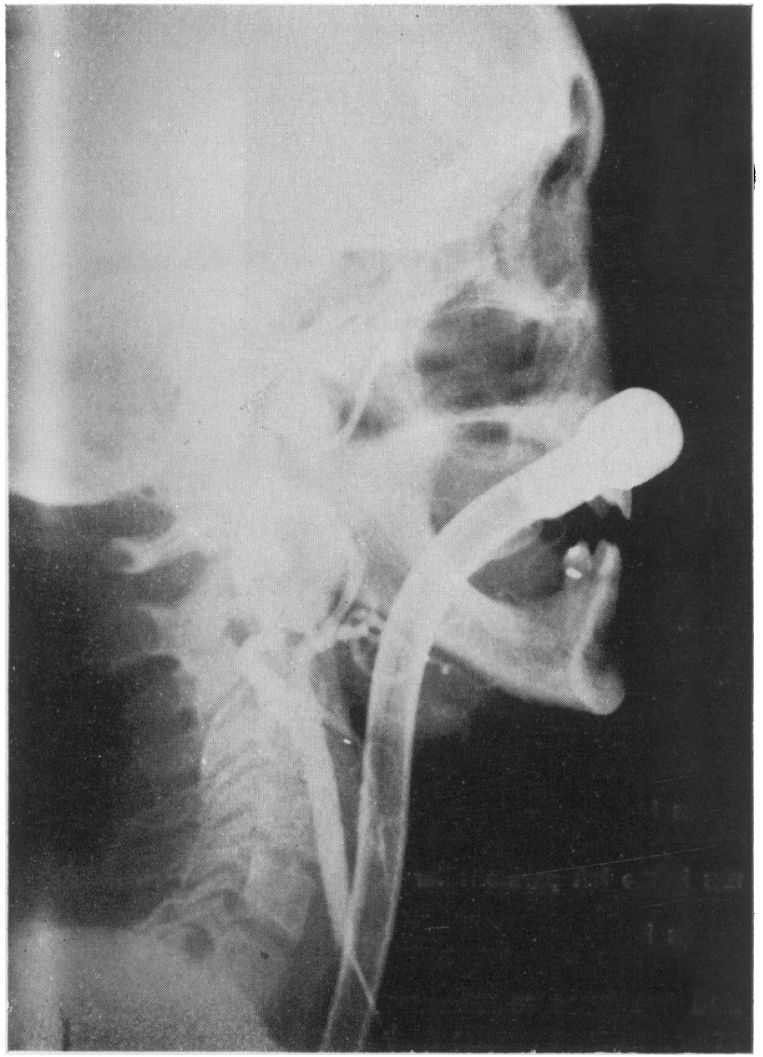

FIG. 2. Arteriogram of the left common carotid artery showing an aneurysm starting $2 \mathrm{~cm}$ distal to the bifurcation and extending to the base of the skull.

at the base of the skull. Surgical exploration of such an aneurysm to confirm the absence of a redundant stump of artery between the aneurysm and skull base is advocated by some in this situation. However, this is debatable and must be weighed against the risks of dislodging emboli and the extensive dissection involved. Successful repair on the base of the skull has been described by Morgan and Robertson (1975). Exposure of the base of the skull in itself may require division of the mandible. The problem of control of the distal vessel has been overcome by means of a balloon catheter (Mengoli, 1969). Hershey (1974) used a variation of this technique by plugging the distal lumen with a tapered hollow catheter, the other end of which was inserted into the carotid artery proximal to the aneurysm and the whole being used as a shunt. The aneurysm was then repaired using the technique of endo-aneurysmorrhaphy first described for popliteal aneurysm by Matas in 1903, the tapered catheter also serving as the splint.

Proximal ligation of an aneurysm has been a simple, standard form of surgical treatment since

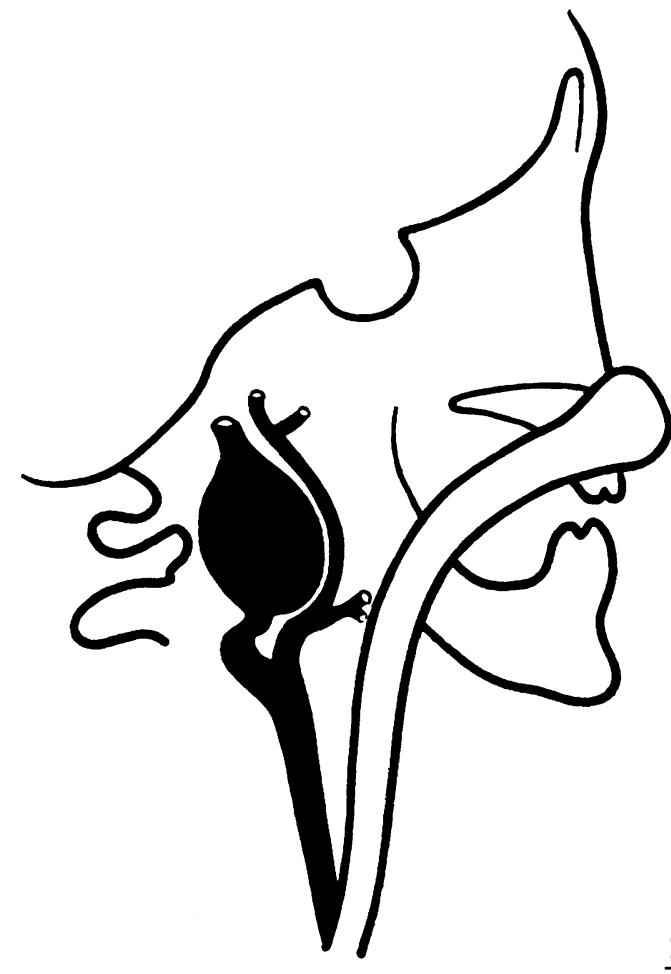

Fig. 3. Tracing of arteriogram.

ancient times, being described by Areteus in the 5 th century AD. Carotid artery ligation was originallyo performed for trauma. Paré ligated a carotid arteryo traumatized in a duel in about 1552 but the patiente developed a monoplegia and died soon after $\overrightarrow{\vec{\sigma}}$ (Watson and Silverstone, 1939). There are severaB cases reported in the late 18th century. Abernethy (1804) ligated a carotid artery damaged by a cow's horn. He first applied temporary occlusion of the carotid artery for some minutes, and only proceedec. to permanent occlusion when there were no il? effects, thus demonstrating his remarkable awareness. of cerebrovascular physiology. However, the patien? died, probably of sepsis, although he obviously developed a contra-lateral neurological deficit before death. The first accurately detailed account of this operation, which was also completely successful was given by David Fleming who performed it an sea on 9th October 1803. The patient was a sailor who had attempted suicide by cutting his throa (Keevil, 1949). In 1805, Sir Astley Cooper per- $-\omega$ formed the first elective ligation of the carotid artery for extra-cranial aneurysm. The patient developed hemiplegia after 8 days and died of sepsis. Three years later Cooper repeated the operation and the patient survived in good health to die from a massivecerebral haemorrhage 13 years later (Cooper, 1836) 
Today, carotid artery ligation, apart from its use in trauma and extra-cranial aneurysm, has a well recognized place in the management of both intracranial aneurysms following subarachnoid haemorrhage and of the rarer condition of arterio-venous fistula in the cavernous sinus.

Cerebral ischaemia with resultant neurological deficit following carotid artery ligation may be immediate, delayed (as in Astley Cooper's first case) or transient (as in the present case report). The exact patho-physiological explanations for these sequelae are not as clear as might be thought, especially in the case of delayed ischaemia. Congenital absence of completion of the circle of Willis has been noted in some post-mortem series as being present in about $30 \%$ of all subjects, and has been blamed as a cause for cerebrovascular ischaemia following carotid artery ligation, since $30 \%$ of unselected ligations develop hemiplegia. However, Nishioka (1966) reviewed 785 cases of unselected ligations with 233 cases of hemiplegia, 35 of whom died and came to post-mortem. In 19 of these, there was no evidence of any anatomical abnormality. In fact Nishioka concluded that the anatomical appearance of the circle of Willis bore little relationship to the functional result. $\mathrm{He}$ also noted that $80 \%$ of the episodes of cerebral ischaemia occurred within $48 \mathrm{hr}$ of ligation, $10 \%$ after 5 days and $5 \%$ after one month. In all his cases, however, there had been recent subarachnoid haemorrhage and these figures may not be entirely relevant to ligation of the carotid artery for other reasons.

The phenomenon of delayed ischaemia is more difficult to explain and, when it occurs, is often related to hypoxia or sudden change in posture. At one time cerebral artery thrombosis due to cephalad promulgation of clot from the site of the ligature was always considered the mechanism for this phenomenon. Dandy (1942) felt this to be the mechanism, but admitted that in several cases that came to post-mortem there was no evidence of thrombosis; he also commented that recurrent episodes of cerebrovascular insufficiency are difficult to explain on this theory. He considered the thrombus to be initiated by damage to the intima of the artery by a narrow ligature, and therefore advocated occlusion of the vessel with a band.

Animal models are not satisfactory to study this problem, since only primates have a semi-vertical posture and, even in this species, the brain tolerates ischaemia better than it does in man. Sengupta et al. (1972), however, have shown that in experiments on the baboon, although local cerebral blood flow was not altered after unilateral carotid artery ligation at normocapnia, there was a gross reduction in the ability of the cerebral vessels to dilate in response to hypercapnia or hypotension. This leads to the hypo- thesis that, after ligation of the carotid artery, blood flow is maintained to the ipsilateral cerebral hemisphere by maximal cerebral vessel dilatation. Dyken (1971) observed that patients who had a pathologically occluded carotid artery were unable to increase their cerebral blood flow whilst breathing $5 \%$ carbon dioxide, unlike normal controls, suggesting that all the vessels in the ipsilateral hemisphere were maximally dilated. Should dilatation then occur in the contra-lateral hemisphere, a 'steal' phenomenon results causing ipsilateral ischaemia, since further dilatation on that side is impossible. This would certainly account for delayed ischaemia, especially when associated with postural or hypoventilating changes. Leech et al. (1974) stress the importance of avoiding hypoxia or hypotension in the postoperative period; one of their patients developed a hemiparesis following a temporary under-ventilation problem during recovery from the general anaesthetic. However, this hypothesis does not properly explain the ability of the cerebral circulation to adapt with time, since, as was apparent in the present case, such adaptation is possible. A possible solution was proposed by Dorrance (1934) when he reviewed several hypotheses including the 'promulgation of thrombus' theory mentioned above. He suggested that eddy currents reflexed by a ligature on the internal carotid artery just distal to the carotid sinus will produce an artificially high distension pressure on the side walls of the sinus. In the event of postural hypotension, afferent impulses from the sinus will be reduced and an inadequate systemic response will result. Being a neurologically based servo-mechanism, adaptation should be possible.

The practical problem facing the surgeon is the selection of patients who can tolerate this procedure before permanent ligation is performed. Should the patient be shown to be intolerant of this procedure the relatively new technique of superficial temporalto-middle cerebral bypass might be performed before ligation (Goldstone, 1977). Abernethy's method of temporary ligation in the conscious subject is the best known, and was used in the present patient. The procedure has been modified by quantitative tests such as monitoring the patient's hand strength, but carries the disadvantage that delayed ischaemia cannot be predicted. Matas (1934) described various techniques based on trials of digital carotid artery compression. Radiographic studies using arteriography with carotid artery compression have been standard for many years. However, these tend to show the anatomical anastomotic situation rather than the functional one, and are often done in vessels recently irritated by subarachnoid haemorrhage, such irritation being increased by the injection of contrast media. Gradual carotid compression was a popular method during the 
1960s, but Brice, Dowsett and Louce (1974) have questioned its value, both in its ability to prevent cerebral ischaemia and to cause thrombosis of berry aneurysms. Recent studies suggest that the most useful information can be obtained by studying the effect of temporary carotid artery occlusion on total or regional cerebral blood flow (CBF). An absolute minimal flow of $18 \mathrm{ml} / 100 \mathrm{~g}$ of brain mass has been defined but the concomitant reduction in blood pressure within the internal carotid artery (ICAP) distal to the point of occlusion, i.e. the back pressure across the circle of Willis, has also been shown to be important, irrespective of blood flow.

The most widely used method of estimating CBF has been the estimation of clearance of gamma activity over the frontal lobes after bolus injection of a radio-active isotope directly into the carotid artery; $2-3 \mu \mathrm{Ci}$ of ${ }^{133} \mathrm{Xe}$ is the usual marker. This requires sophisticated equipment, whereas pressure measurement such as used by the present authors is much more readily available.

Oller, Gee and Kingsley (1976) describe a comparison of ICAP monitoring with the relatively non-invasive technique of ocular pneumo-ophthalmography. In this method, a measured pressure is applied to the eyball and increased to the point where retinal artery blood flow ceases. This pressure (OPG) reflects retinal arterial pressure, and related well to ICAP before and after carotid occlusion. An OPG of $80 \mathrm{mmHg}$ correlated with an ICAP of $60 \mathrm{mmHg}$, and in their study based on 4 patients, Oller et al. concluded that an OPG of $80 \mathrm{mmHg}$ after temporary carotid artery occlusion indicated that permanent ligation was safe.

Leech et al. (1974) have produced the most comprehensive practical guide to the safety of internal carotid artery ligation using the combined measurements of CBF and ICAP after temporary occlusion. In a series of 20 patients they concluded that in all circumstances a CBF of $40 \mathrm{ml} / \mathrm{min} / 100 \mathrm{~g}$ of brain mass indicated that ligation was always safe, and that $20 \mathrm{ml}$ was never safe. In the range between these two extremes, ligation was still safe in the reduction of CBF was less than $25 \%$ of the pre-ligation CBF and that this could be extended to $35 \%$ provided that stump ICAP (i.e. the back pressure across the circle of Willis) was at least $60 \mathrm{mmHg}$ in the normotensive individual. Leech et al. recommended that if pressure measurement alone was the only facility available, then a minimal level of stump ICAP of $60 \mathrm{mmHg}$ should be accepted as safe in the normotensive patient. However, in $10 \%$ of their patients in which stump ICAP remained higher than 60 $\mathrm{mmHg}, \mathrm{CBF}$ fell below the critical level of $20 \mathrm{ml}$.

In the present patient, stump ICAP fell to 65 $\mathrm{mmHg}$. This, supported by the patient's subjective and objective tolerance to temporary occlusion, was considered safe. No evidence of early ischaemia developed but on mobilization she developed signs of delayed ischaemia. Obviously her CBF adapted to postural response after a time, but the mechanism by which this occurs is by no means clear.

\section{References}

Abernethy, J. (1804) Surgical Observation, p. 103. Longman \& Rees, London.

Alexander JR, E., Wigser, S.M. \& Davis, C.H. (1964) Bilateral extra-cranial aneurysms of the internal carotid arteries. Journal of Neurosurgery, 25, 437.

Beall, A.C., Crawford, E.S., Cooley, D. \& De Bakey, E. (1962) Extra-cranial aneurysms of the carotid artery. Postgraduate Medicine. Milwaukee, etc., 32 (2), 93.

Bergentz, S.E., Ericsson, B.F., Linell, F. \& Olivecrona, H. (1976) Bilateral fibromuscular hyperplasia in the internal carotid arteries with aneurysm formation. Acta chirurgica scandinavica, 142, 501.

Brice, J.G., DowseTt, D.J. \& Louce, R.D. (1974) Haemodynamic effects of carotid artery stenosis. British Medical Journal, 2, 1363.

Carmichael, R. (1950) The pathogenesis of non-inflammatory cerebral aneurysms. Journal of Pathology and Bacteriology, 62, 1.

Colin, J.F. \& Ellis, H. (1971) Fusiform aneurysms of the brachial artery in a child treated by proximal ligation. Postgraduate Medical Journal, 47, 323.

COOPER, A. (1836) Account of the first successful operation performed on the common carotid artery for aneurysm in the year 1808 with post-mortem examination in the year 1821. Guy's Hospital Reports, 1, 53.

DANDY, W.E. (1942) Results following ligation of the internal carotid artery. Archives of Surgery, 45, 521.

DorRanCE, G.M. (1934) Ligation of the great vessels of the neck. Annals of Surgery, 99, 721.

DYKEN, M.L. (1971) Intra-cranial 'steal' in complete oc-60 clusion of the internal carotid artery. European Neurology, 6, 301.

GoldsTONE, J. (1977) In: Vascular Surgery (Ed. Rutherford, R.B.), p. 1137. W. B. Saunders Co., Philadelphia.

Hershey, F.B. (1974) Operation for aneurysm of the internal carotid artery high in the neck. Angiology, 25 (1), 24.

KeEviL, J.J. (1949) David Fleming and the operation of ligation of the carotid artery. British Journal of Surgery, 37, 92.

Leech, P.J., Miller, J.D., Fitch, W. \& Barker, J. (1974). Cerebral blood flow, internal carotid artery pressure and the EEG as a guide to the safety of carotid artery ligation. Journal of Neurology, Neurosurgery, and Psychiatry, 37, 854.

MatAs, R. (1903) An operation for the radical cure of aneurysm based upon arteriorrhaphy. Annals of Surgery, 37, 161.

Matas, R. (1934) Discussion of a paper by Elkin and Campbell. American Journal of Surgery, 24, 692.

MENGoli, L.R. (1969) Aneurysmorrhaphy of the internal carotid artery, utilizing intra-luminal distal control. American Journal of Surgery, 117, 397.

Morgan, H. \& Robertson, J.T. (1975) Proceedings of the Society of British Neurological Surgeons. Journal of Neurology, Neurosurgery and Psychiatry, 38 (4), 409.

NishiokA, H. (1966) Report on the co-operative study of intra-cranial aneurysms. Journal of Neurosurgery, 25, 660.

Oller, D.W., Gee, W. \& Kingsley, J.R. (1976) Treatment for high extra-cranial internal carotid aneurysm. American Surgeon, 42 (5), 311.

PARÉ, A. (1634) The Works of that Famous Chirurgian Ambroise Pare translated out of the Latin and compared with the French hy Thomas Johnson. Reynolds, London. 
Rydell, J.R. \& JenNings, W.K. (1956) The surgical treatment of carotid aneurysms in the neck. Western Journal of Surgery, Obstetrics and Gynecology, 64, 385.

Sengupta, D., Harper, A.M., Deshmukh, V.D., Rowan, J.O. \& JENNETT, W.B. (1972) Effect of carotid artery ligation on the carbon dioxide response in the baboon. European Neurology, 6, 369.
Thompson, J.E. \& Austin, D.J. (1957) Surgical management of cervical carotid aneurysms. Archives of Surgery, 74, 80. WATson, W.L. \& Silverstone, S.M. (1939) Ligature of the common carotid artery in cancer of the head and neck. Annals of Surgery, 109, 1.

WINSLOw, N. (1926) Extra-cranial aneurysms of the internal carotid artery. Archives of Surgery, 13, 689. 